\title{
A Bibliometric Analysis of Coronavirus Research in Gulf Cooperation Council Countries
}

\author{
https://doi.org/10.3991/ijoe.v17i13.27367 \\ Ali Mustafa Qamar( $\left.{ }^{\bowtie}\right)$, Rehan Ullah Khan, Suliman A. Alsuhibany \\ Qassim University, Buraydah, Saudi Arabia \\ al.khan@qu.edu.sa
}

\begin{abstract}
COVID-19 was declared a pandemic by World Health Organization in March 2020. Since then, it has attracted the enormous attention of researchers from around the world. The world has gone through previous instances of corona-viruses such as Severe Acute Respiratory Syndrome and Middle Eastern Respiratory Syndrome. Nevertheless, none was of these were of this serious nature as COVID-19. In this research, we carry out a bibliometric analysis of coronavirus research using the Scopus database. However, we restricted ourselves to the Gulf Cooperation Council countries, comprising Bahrain, Kuwait, Oman, Qatar, Saudi Arabia, and the United Arab Emirates. The analysis was performed using Biblioshiny software. We analyzed 4288 articles written by 24226 researchers from 1994 till 2021, published in 1429 sources. The number of authors per publication is 5.65. A bulk of the research (more than 68\%) appeared in the form of articles. More than $43 \%$ of the publications appeared in 2020 and more than $44 \%$ in 2021 . Saudi Arabia appears the most-cited country, followed by Qatar. Journal of Infection and Public Health published the most number of papers, whereas New England Journal of Medicine is the most-cited one. Memish, Z.A. wrote the maximum number of papers. The top source, according to the H-index, is the Journal of Virology. Furthermore, the two most prolific universities are King Saud University and King Abdulaziz University, both from Saudi Arabia. The research uncovered deep learning as a niche theme used in recent publications. The research landscape continues to alter as the pandemic keeps on evolving.
\end{abstract}

Keywords-COVID-19, coronavirus, bibliometric, Gulf Cooperation Council

\section{Introduction}

Since early 2020, the entire world is grappling with the pandemic of coronavirus disease 2019 (COVID-19). As of Aug 22, 2021, there have been more than 204 million cases of COVID-19 and more than four million deaths $(2.11 \%$ of the total cases) worldwide. Similarly, more than 183 million people $(89.8 \%)$ have recovered from this disease [1]. The worst affected countries are the USA, India, Brazil, Russia, France, UK, Mexico, Peru, Italy, and Columbia in terms of the total cases and total deaths. COVID-19 was first found in Wuhan, China, in December 2019. It was declared as a pandemic by the World Health Organization (WHO) on Mar 11, 2020. 
Bibliometric studies approach the given problem by analyzing the related publications from different databases such as PubMed, Scopus, and Web of Science. Two coronavirus outbreaks occurred in the last 20 years, namely, the Severe Acute Respiratory Syndrome (SARS) in 2002-2003 in China, and the Middle East Respiratory Syndrome (MERS) in 2012 in the Middle East. However, they have not been extensively studied through bibliometric research. On the contrary, COVID-19 has been studied in detail during 2020 and 2021. Guleid et al. [2] conducted a bibliometric analysis of COVID-19 in Africa.

Similarly, Zyoud [3] tried to find the Arab world's contribution to COVID-19 research through a bibliometric study. However, we know of no previous work where the researchers have studied the coronavirus from a bibliometric perspective in the Gulf Cooperation Council (GCC) countries. This paper discusses a thorough bibliometric analysis of coronavirus research in GCC using the Scopus database. GCC consists of six countries, Bahrain, Kuwait, Oman, Qatar, Saudi Arabia, and United Arab Emirates (UAE). It was founded in 1981 and comes at seventh place in the world in terms of total GDP and the per capita GDP. We analyzed the growth of the scientific production related to coronavirus specific to the GCC region. We found the most active countries in research; most-cited authors, papers, and countries, collaboration networks of countries, researchers, and institutions.

The rest of the paper is organized as follows: Section 2 presents a literature review, whereas the methodology is discussed in Section 3. The results are discussed in Section 4 , and the paper is concluded in Section 5 along with providing some future works.

\section{$2 \quad$ Literature review}

Gong et al. [4] performed a bibliometric analysis on the early research on COVID19. They studied 1500 research papers retrieved from PubMed and China National Knowledge Infrastructure from Jan 1, 2020, till Mar 8, 2020. They found that the number of Chinese articles was more than 2.5 times compared to those written in the English language, especially after mid-Feb 2020. One of the reasons could be that China was the most affected country during the early months of the pandemic. A strong collaboration between the USA and China was observed, whereas Saudi Arabia was the only GCC country that appeared in the collaboration network. Moreover, a consolidated knowledge map including diagnosis, treatment, prevention and control, and the prognosis was also presented.

Zyoud [3] examined the contribution of the 22 countries of the Arab world for COVID-19 research using bibliometric analysis. Six thousand one hundred thirty-one documents were analyzed, amounting to $4.26 \%$ of the global research. More than $35 \%$ were published by Saudi Arabia, followed by Egypt $(20.78 \%)$ and UAE $(11.73 \%)$. International Journal of Environmental Research and Public Health was found to be the most sought-after journal. Four of the ten most productive institutions belong to Saudi Arabia, with King Saud University being at the top with 429 publications (7\% of the total). 
In [5], the researchers approached coronavirus from a Scientometrics point of view. They analyzed 50 years (1970-2019) of articles related to coronaviruses. The data included 5128 articles obtained from the Web of Science.

Zyoud [6] conducted a bibliometric analysis of MERS coronavirus and analyzed 883 publications written in four years, between 2012 and 2015. Most of the articles are written by the USA (319) and Saudi Arabia (113). Farooq et al. [7] focused only on Web of Science publications related to COVID-19, and that too for 2019 and 2020. They analyzed 6694 papers, out of which 324 were published in 2019 and got 2105 citations, whereas 6370 publications were observed in the first six months of 2020, gathering 37,965 citations. USA and China were found to be the most productive countries. However, two Chinese universities, Huazhong University of Science and Technology and Wuhan University, published the highest number of papers and collectively got more than 10000 citations.

Kagan et al. [8] analyzed more than 45 million publications from the last 20 years to uncover the scientometrics trends of earlier coronaviruses, including SARS and MERS. Most of the research originated in China and the USA, as found by many of the related researches. Lou et al. [9] performed a bibliometric analysis of COVID-19 and worked on 183 publications retrieved from the PubMed database. They searched for the keywords "COVID-19" from January 14, 2020, to March 1, 2020. Nevertheless, they only analyzed the title, corresponding author, language, publication time while omitting the abstracts. COVID-19 Open Research Dataset (CORD-19) is freely available for researchers [10]. It has more than 52,000 publications, where more than 41,000 are full-texts.

Zyoud and Al-Jabi [11] performed a preliminary analysis of COVID-19 based on a bibliometric study. They studied more than 19,000 papers published between Dec 2019 and June 19, 2020, which are available in the Scopus database. According to their research, the USA published the most papers, followed by China, Italy, and the UK. British Medical Journal was the most productive in terms of papers (522 or $2.74 \%$ of the papers), and Huazhong University of Science and Technology, China, was the most prolific institution for COVID-19 research and published more than $2 \%$ of all research articles.

Ho et al. [12] conducted a bibliometric analysis of 674 publications related to ten psychology-related Web of Science categories in the social citation index. $46 \%$ of the articles are related to clinical psychology. Similarly, $24 \%$ of the publications are Editorials and letters.

Chahrour et al. [13] recommended increasing the research activity related to COVID-19. PubMed and WHO database for COVID-19 publications were explored and only 564 publications were analyzed. Surprisingly, $67 \%$ of the papers were written by China. The study was conducted during the earlier days of the pandemic.

Luo [14] gave a data-driven estimation regarding the end of COVID-19 in various countries. The proposed epidemic model was based on Regression. His research predicted on April 26, 2020 that the pandemic would peak on May 24, 2020, in Saudi Arabia and end entirely by June 1,2020. However, COVID-19 cases keep coming today, and multiple peaks have been observed in various countries. 


\section{$3 \quad$ Methodology}

This research employs a bibliometric method to analyze the available literature on different types of coronavirus. However, we focused only on the six countries of the GCC. The key approach is founded on the analysis and measurement of the scientific literature related to coronavirus. Scopus was chosen as the search engine since it is one of the main sources of scientific literature. The following search query was executed in the Article title, Abstract, Keywords field.

$\mathrm{T} 1=\mathrm{TITLE}-\mathrm{ABS}-\mathrm{KEY}$ (coronavirus OR corona AND virus OR covid OR sars OR "Severe Acute Respiratory Syndrome" OR MERS OR "MIDDLE EAST RESPIRATORY SYNDROME" OR covid-19 OR sars-cov-2). Six GCC countries are used as the country keys.

This article's data were retrieved from Scopus on Aug 5, 2021, and contained 4288 publications. The earliest publications are from 1994, and the latest ones are published in 2021. The papers were published in 1429 sources. The sources include articles, book chapters, conference papers, data papers, letters, notes, editorials, reviews, and short surveys. We perform a quantitative analysis and present our research findings in the form of graphs and network models.

R-studio is an IDE for R, a programming language used for statistical computation. We used Biblioshiny, an app for bibliometrix, which is, in turn, an R-studio tool to perform science mapping analysis. It summarizes the data in the form of total authors, total citations, single-author, and multiple-author publications.

\section{$4 \quad$ Experiments and results}

Here, we provide the results obtained from biblioshiny. The average citation per document is 16.29 . Furthermore, the number of authors is 24226 , where the singleauthored documents were just 258 , and the rest were multi-authored ones. The average number of documents per author was 0.177 . Table 1 provides an insight into the various types of available documents. Most of the documents are articles (2917, $68.03 \%)$, followed by reviews $(769,17.93 \%)$.

Figure 1 shows the top 20 most relevant sources. The most common source is the Journal of Infection and Public Health, where 102 documents were published. The next one on the list is the International Journal of Environmental Research, containing 91 papers. Similarly, 83 papers were published in the International Journal of Infectious Diseases. Only six journals published more than 50 papers. 
Table 1. Document type-wise distribution in Coronavirus research

\begin{tabular}{|l|c|c|}
\hline \multicolumn{1}{|c|}{ Document types } & No. of records & Percentage \\
\hline Article & 2917 & 68.03 \\
\hline Article in press & 0 & 0.00 \\
\hline Book & 0 & 0.00 \\
\hline Book Chapter & 18 & 0.42 \\
\hline Conference paper & 83 & 1.94 \\
\hline Conference Review & 0 & 0.00 \\
\hline Data paper & 3 & 0.07 \\
\hline Editorial & 121 & 2.82 \\
\hline Erratum & 3 & 0.07 \\
\hline Letter & 270 & 6.30 \\
\hline Note & 80 & 1.87 \\
\hline Review & 769 & 17.93 \\
\hline Short survey & 24 & 0.56 \\
\hline
\end{tabular}

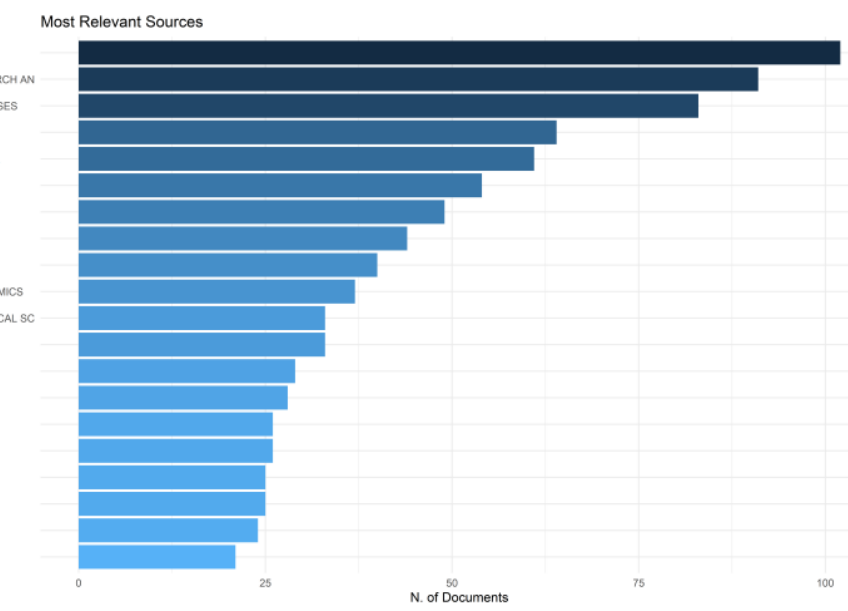

Fig. 1. Top 20 most relevant sources

Figure 2 shows the top 20 most cited sources. The topmost cited source is the New England Journal of Medicine, containing 4025 citations. Similarly, 3633 citations are for the papers published in the Lancet journal. One can notice that only four journals were able to get more than 2000 citations. 


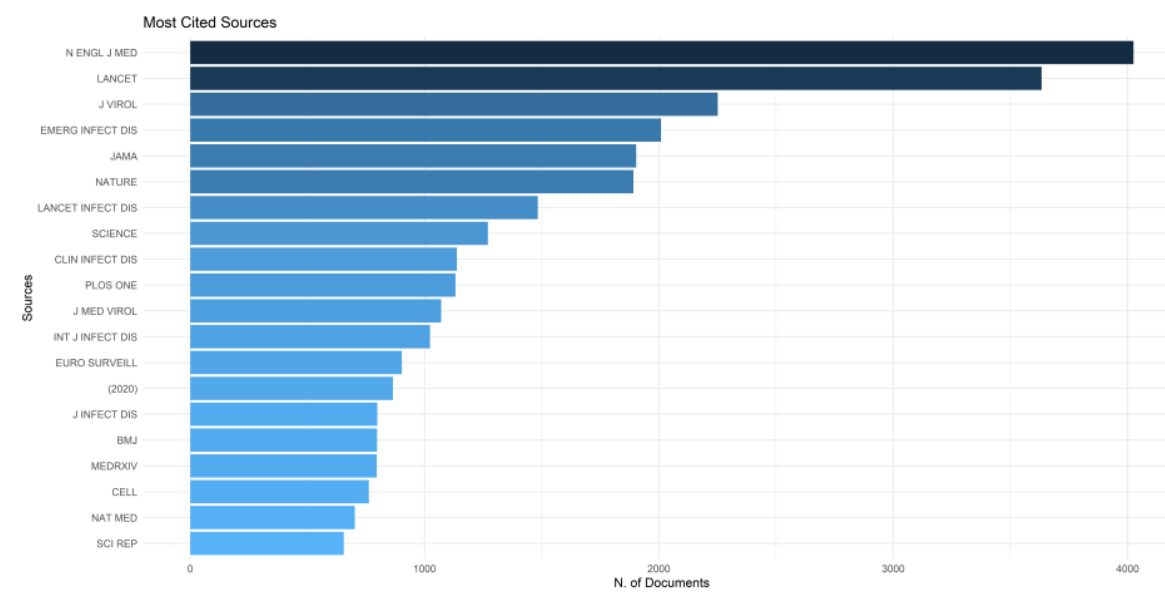

Fig. 2. Top 20 most cited sources

Figure 3 depicts the annual scientific production. It can be seen that the publications increased rapidly in the year 2020 (1853 or 34.69\%) and 2021 (1878) with the onset of COVID-19. The publications increased to 79 in 2014, 99 in 2016, and slightly decreased to 96 in 2019.

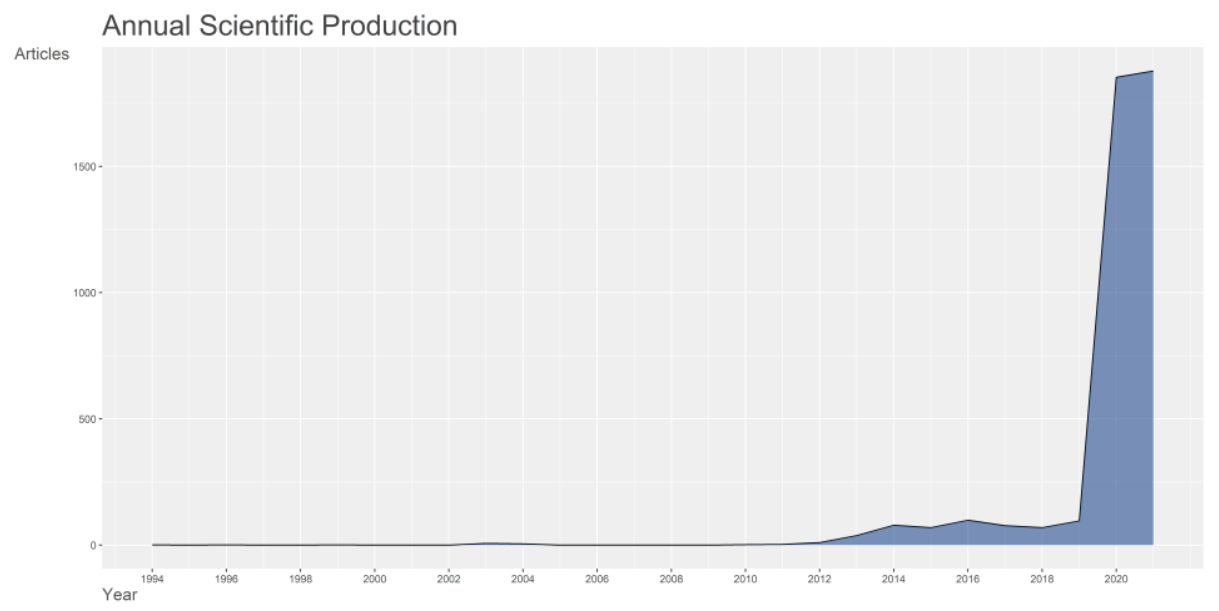

Fig. 3. Annual Scientific Production from 1994 till 2021

Figure 4 shows the average article citations per year between 1994 and 2021. This graph is very interesting, and one can observe two peaks, one in 2012 and the other in 2021. The first peak coincided with the discovery of MERS and the second one corresponds to the second year of the COVID-19 pandemic. In both of these years, the average article citations increased to more than 45 . 


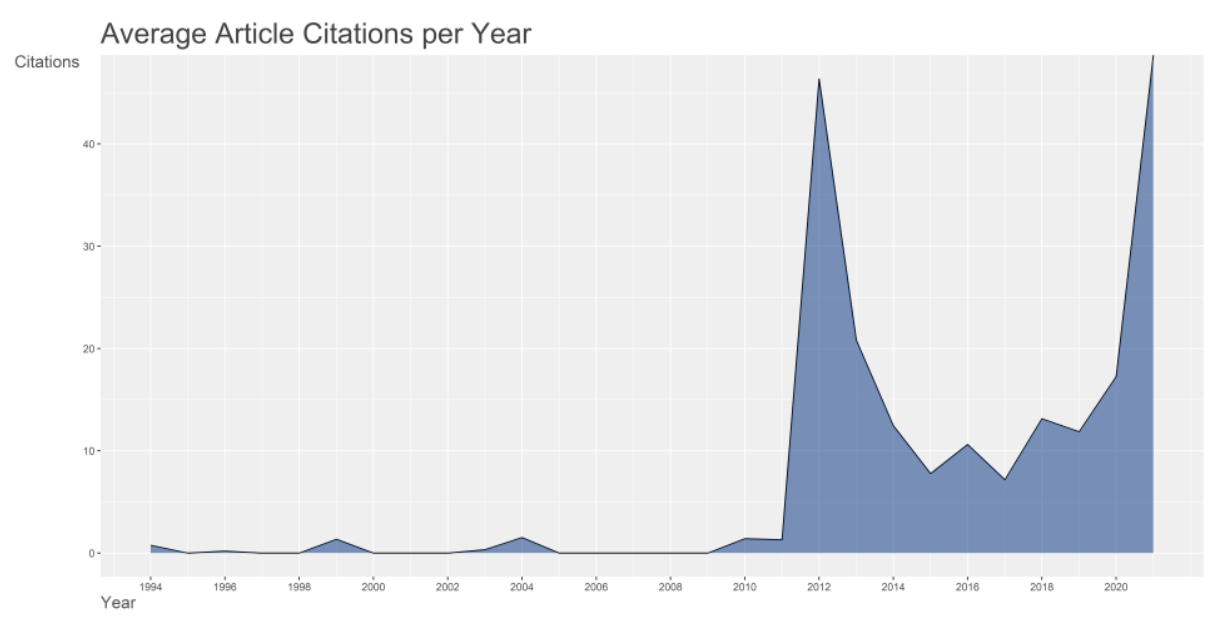

Fig. 4. Average article citations per year

Table 2 shows the most frequent words found in the Abstracts. As expected, the most common word is covid- which is found 10127 times. The other two most common words are patients and coronavirus, having 4773 and 3579 occurrences, respectively. In Titles of the papers, the most common words are covid-, coronavirus, and pandemic. This depicts a trend that while the word patients are commonly used in the Abstracts, it is generally avoided in the Titles. Similarly, the most common keywords supplied by the researchers are covid-19, sars-cov-2, and coronavirus.

Table 2. The most frequent words found in the Abstracts

\begin{tabular}{|l|c|}
\hline \multicolumn{1}{|c|}{ Most frequent words } & Frequency \\
\hline covid- & 10127 \\
\hline patients & 4773 \\
\hline coronavirus & 3579 \\
\hline pandemic & 3384 \\
\hline disease & 3235 \\
\hline study & 3184 \\
\hline health & 2764 \\
\hline sars-cov- & 2739 \\
\hline respiratory & 2619 \\
\hline infection & 2563 \\
\hline
\end{tabular}

Figure 5 shows the three-field plot. The authors are on the left side of the figure, whereas the countries are in the middle, and the affiliations are on the right side. Among the GCC countries, only Saudi Arabia and Qatar appear in the middle. Similarly, most of the affiliations are related to Saudi Arabian universities, as could be seen on the right side of the figure. Nevertheless, some universities from other GCC countries appear as well, such as the University of Sharjah and the United Arab Emir- 
ates university from UAE, and Qatar University from Qatar. This figure shows that most of the publications are written by Halwani, R., Mem-ish, Z.A., Arabi, Y.M, and Al-Tawfiq, J.A., which have a strong relationship with Saudi Arabia, France, and the USA.

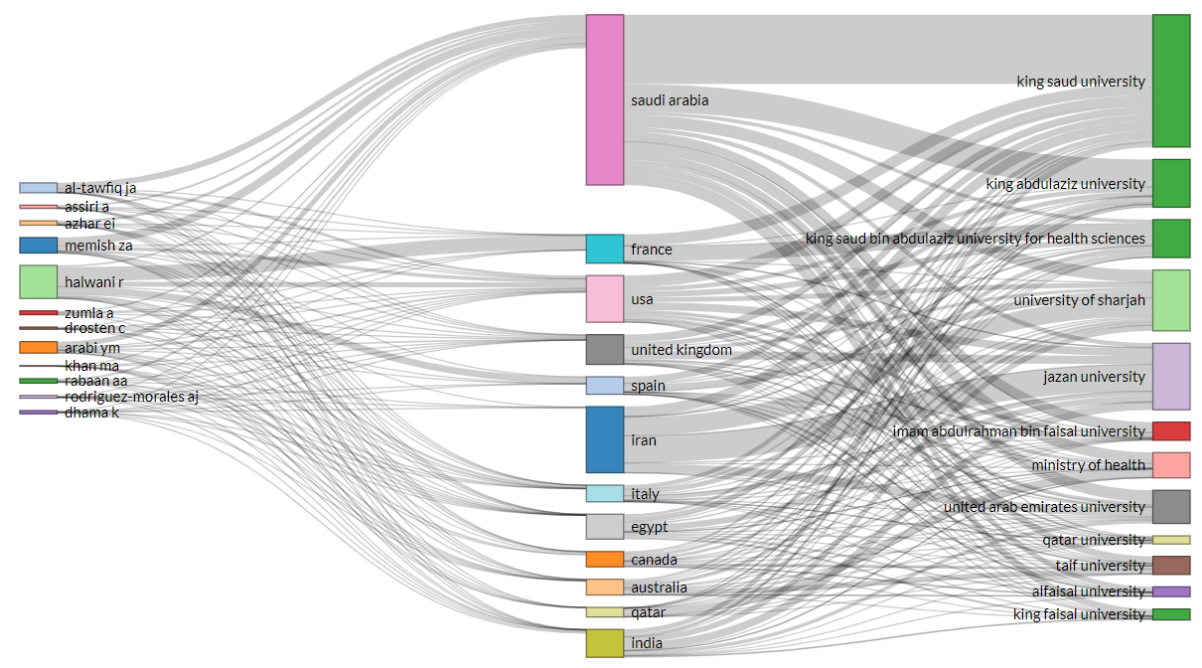

Fig. 5. Three-field plot for researchers, countries, and institutions

Figure 6 shows the most-cited countries. While Saudi Arabia has 23150 citations, Qatar has got only 2299 citations. On the other hand, Kuwait, Oman, and Bahrain have 706, 661, and 140 citations. Average article citations have also been analyzed. Saudi Arabia, Qatar, Kuwait, Oman, and Bahrain have an average article citation of $15.31,9.42,7.06,5.90$, and 4.38.

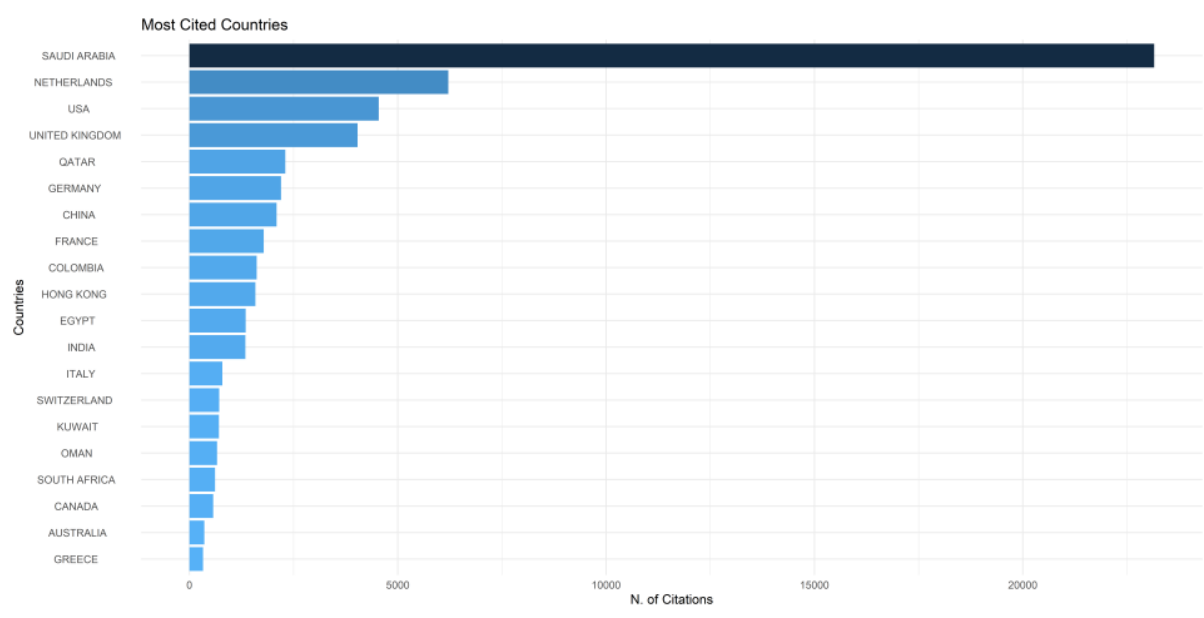

Fig. 6. Top 20 most cited countries 
Moreover, Figure 7 shows the top 20 countries according to the number of citations per year. The Netherlands is at the top with 326.95 average article citations per year. Only one country from GCC, Saudi Arabia, appears in the list at number 19, with 15.31 citations per year.

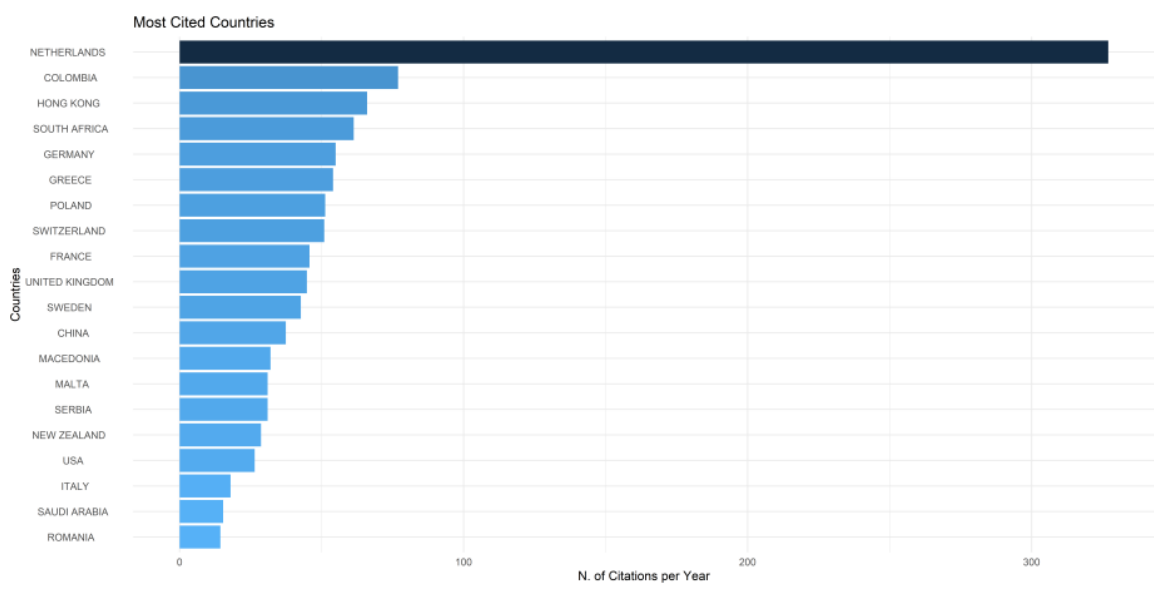

Fig. 7. Top 20 most cited countries per year

Figure 8 shows the most relevant authors in terms of the number of publications. Only Memish, Z.A., and Al-Tawfiq, J.A. were able to publish more than 100 papers.

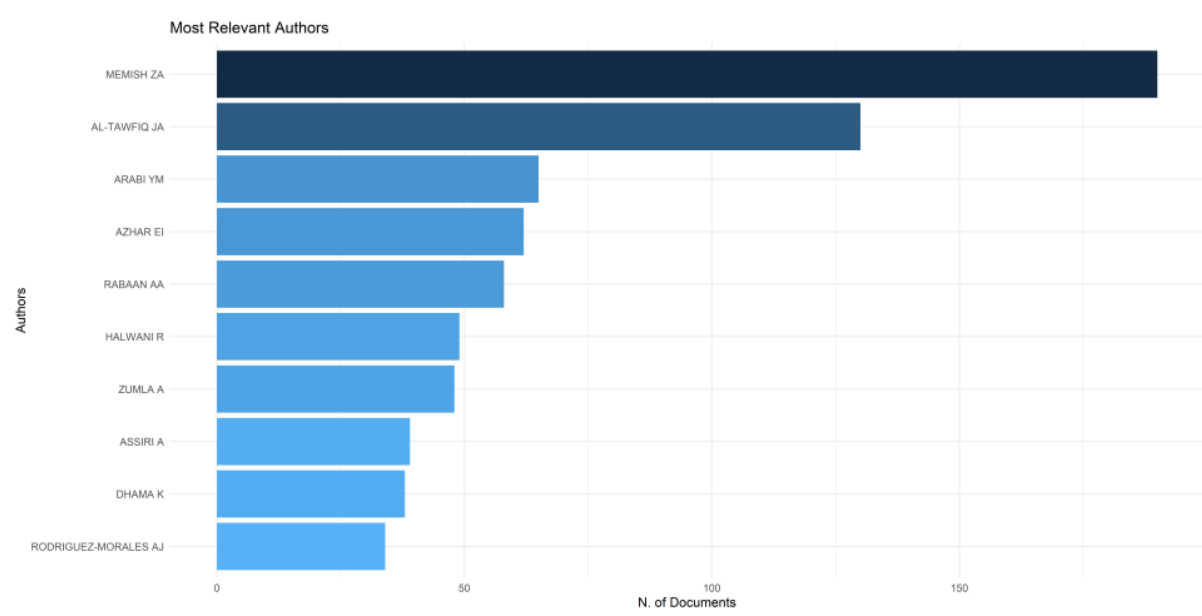

Fig. 8. Top ten most relevant authors

Figure 9 shows the analysis of author productivity while employing Lotka's law. 13414 out of 24226 authors (55.4\%) published only a single article related to coronavirus. Similarly, $20.3 \%$ wrote two articles, and $4.4 \%$ wrote three articles. 


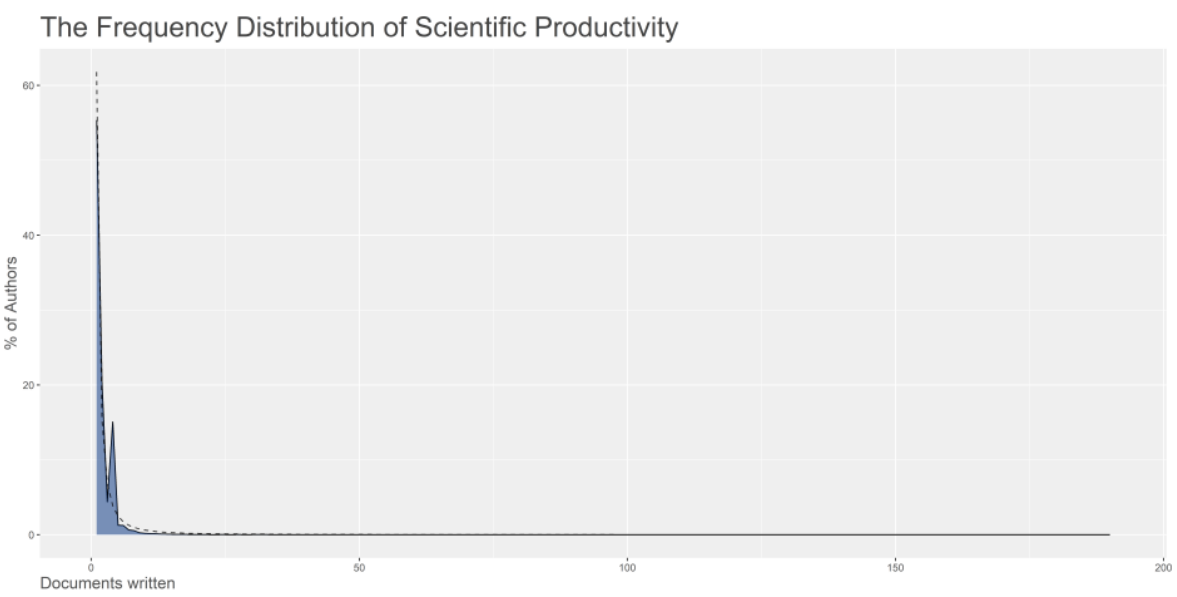

Fig. 9. Frequency distribution of authors' productivity

Global citations help to find the number of citations received by a document from other documents present in the data set. The top ten most cited papers are given in Table 3. The most cited paper is Isolation of a Novel Coronavirus from a Man with Pneumonia in Saudi Arabia by Zaki et al. [15] and appeared in the New England Journal of Medicine in Nov 2012. It has 2864 citations. This coincided with the discovery of MERS coronavirus.

Furthermore, the second most cited paper is The continuing 2019-nCoV epidemic threat of novel coronaviruses to global health — The latest 2019 novel coronavirus outbreak in Wuhan, China by Hui et al. [16] and published in International Journal of Infectious Diseases in Feb 2020, one month before WHO declared COVID-19 as a pandemic. This paper has been cited 1227 times and has the highest total citations (TC) per year (613.5) and the highest normalized TC. It is pertinent to mention that Memish, Z.A. is also one of the authors of this paper.

Table 3. Top ten most cited papers

\begin{tabular}{|l|c|c|c|c|}
\hline \multicolumn{1}{|c|}{ Paper } & Journal & $\begin{array}{c}\text { Total Citations } \\
\text { (TC) }\end{array}$ & $\begin{array}{c}\text { T.C. per } \\
\text { Year }\end{array}$ & $\begin{array}{c}\text { Normalized } \\
\text { T.C. }\end{array}$ \\
\hline Zaki et al [15]. (2012) & NEW ENGL J MED & 2864 & 286.4 & 6.8648 \\
\hline Hui et al. [16]. (2020) & INT J INFECT DIS & 1227 & 613.5 & 71.0665 \\
\hline Raj et al. [17]. (2013) & NATURE & 1070 & 118.8889 & 6.4244 \\
\hline $\begin{array}{l}\text { Rodriguez-Morales et al. } \\
\text { [18]. (2020) }\end{array}$ & TRAVEL MED INFECT DIS & 900 & 450 & 52.127 \\
\hline Assiri et al. [19]. (2013) & LANCET INFECT DIS & 827 & 91.8889 & 4.9654 \\
\hline Assiri et al. [20]. (2013) & NEW ENGL J MED & 779 & 86.5556 & 4.6772 \\
\hline Zumla et al. [21]. (2016) & NAT REV DRUG DISCOV & 775 & 129.1667 & 14.5893 \\
\hline Alhazzani et al. [22]. (2020) & INTENSIVE CARE MED & 697 & 348.5 & 40.3695 \\
\hline De Groot et al. [23]. (2013) & J VIROL & 683 & 75.8889 & 4.1008 \\
\hline $\begin{array}{l}\text { Van Bohemeen et al. [24]. } \\
\text { (2012) }\end{array}$ & mBio & 566 & 56.6 & 1.3567 \\
\hline
\end{tabular}


Figure 10 shows the Source impact for various publication venues in terms of the Hirsch (h) index. It is an author's (or journal's) number of published articles (h), each cited in other papers at least $h$ times. The highest H-index (30) is exhibited by the Emerging Infectious Diseases journal, which started in 2013. Coronavirus is a viral disease, which is perhaps why the top four journals are related to infectious diseases. Similarly, the International Journal of Infectious Diseases comes second with a value of 24, followed by Travel Medicine and Infectious Disease having an H-index of 21. The list also includes two Saudi journals, Saudi Journal of Biological Sciences and Saudi Medical Journal, with H-indices of 9 and 8 . Eleven journals have an h-index greater than or equal to 10 .

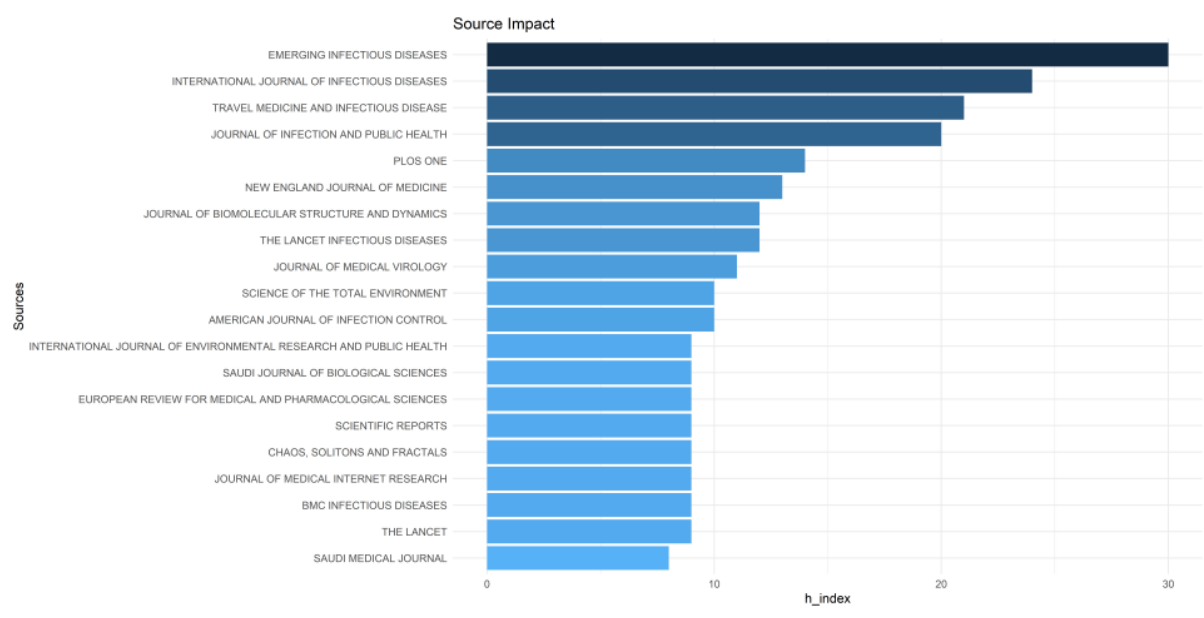

Fig. 10. Top 20 sources according to the H-index

Figure 11 depicts the most relevant affiliations. The maximum number of publications (746) is by King Saud University, Saudi Arabia, whereas King Abdulaziz University, Saudi Arabia, published 635 papers. Similarly, King Saud Bin Abdulaziz University for Health Sciences, also from Saudi Arabia, published 320 papers. Among the top 10 universities, two belong to UAE (University of Sharjah and United Arab Emirates University), and one is from Qatar (Qatar University). Seven universities published more than 200 papers.

Figure 12 shows the Multiple Country Publications (MCP) and Single Country Publications (SCP) for various countries while considering the corresponding author. Saudi Arabia has the largest MCP (735) as well as SCP (777). The following country on the list is Qatar having 145 for MCP and 99 for SCP. The MCP ratio, defined as the ratio of Multiple Country Publications to the total publications for Saudi Arabia, Qatar, Oman, Kuwait, and Bahrain, is 0.486, 0.406, 0.384, 0.490, and 0.375, respectively. These ratios show that the researchers in Kuwait and Saudi Arabia favored MCP greatly compared to the other GCC countries. 


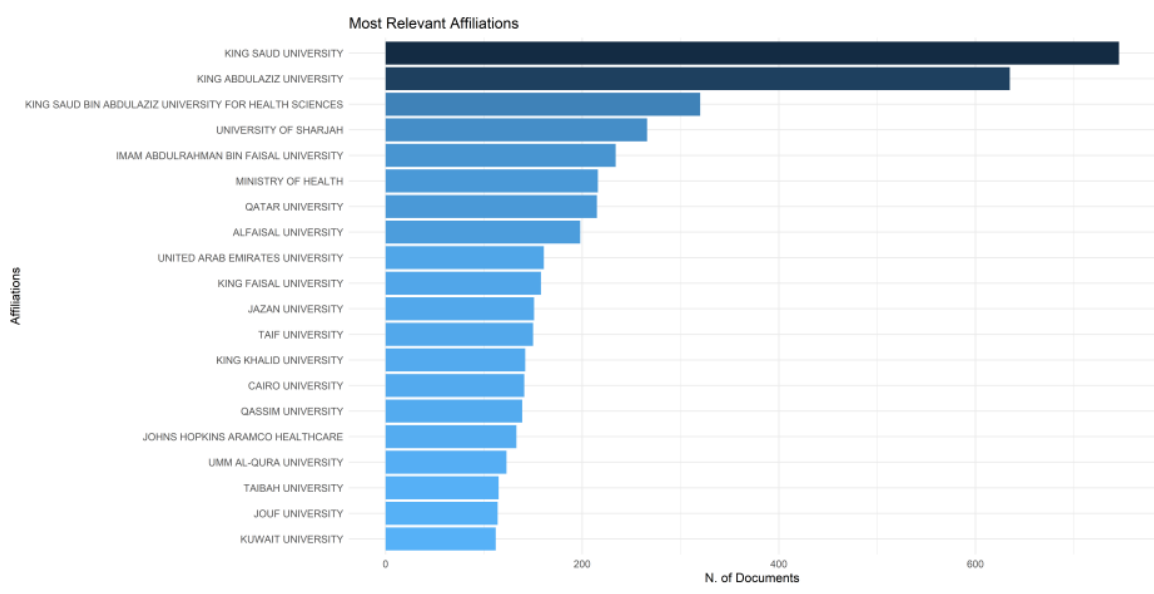

Fig. 11. Top 20 most relevant affiliations

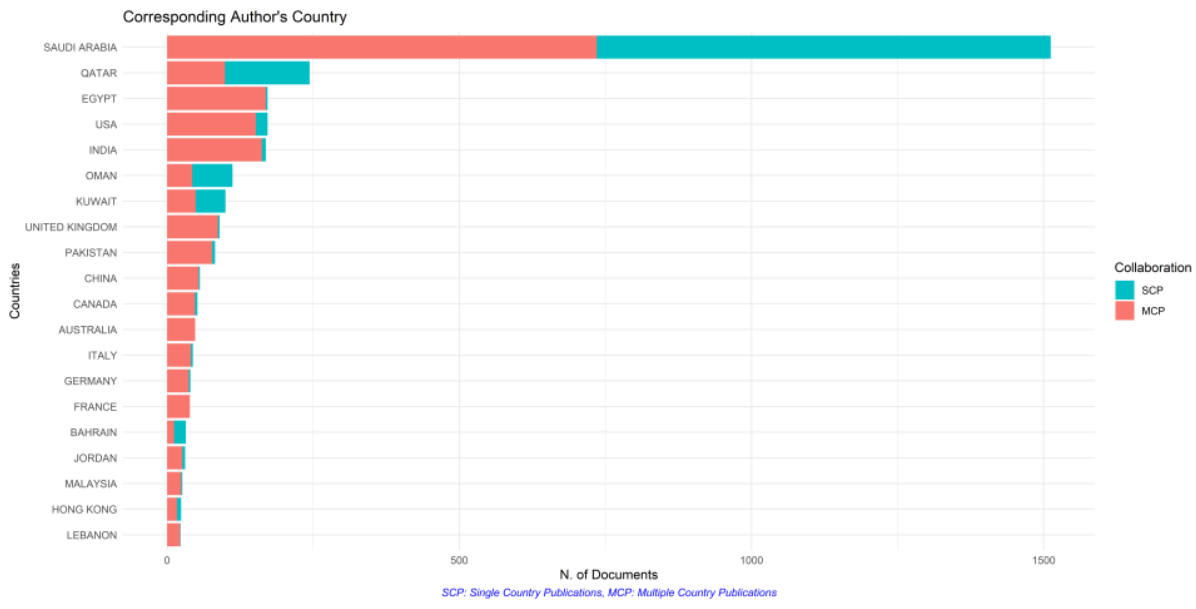

Fig. 12. Top 20 Multiple Country Publications and Single Author Publications

Figure 13 shows the collaboration network for the most active countries. Two collaborative groups are identified as represented in red and blue colors. Bigger rectangles and denser links show strong contributions. The most active collaborations are between Saudi Arabia and the USA, Egypt, India, China, and Pakistan. Saudi Arabia mainly collaborates with Bahrain and Kuwait from the GCC.

Similarly, Saudi Arabia also collaborates with the USA, Egypt, China, India, and Pakistan. In contrast, United Kingdom collaborates with countries such as Oman, Qatar, Germany, Italy, Switzerland, France, Netherlands. It can be observed that more countries are found in Group 2 (represented in blue color) compared to Group 1. 


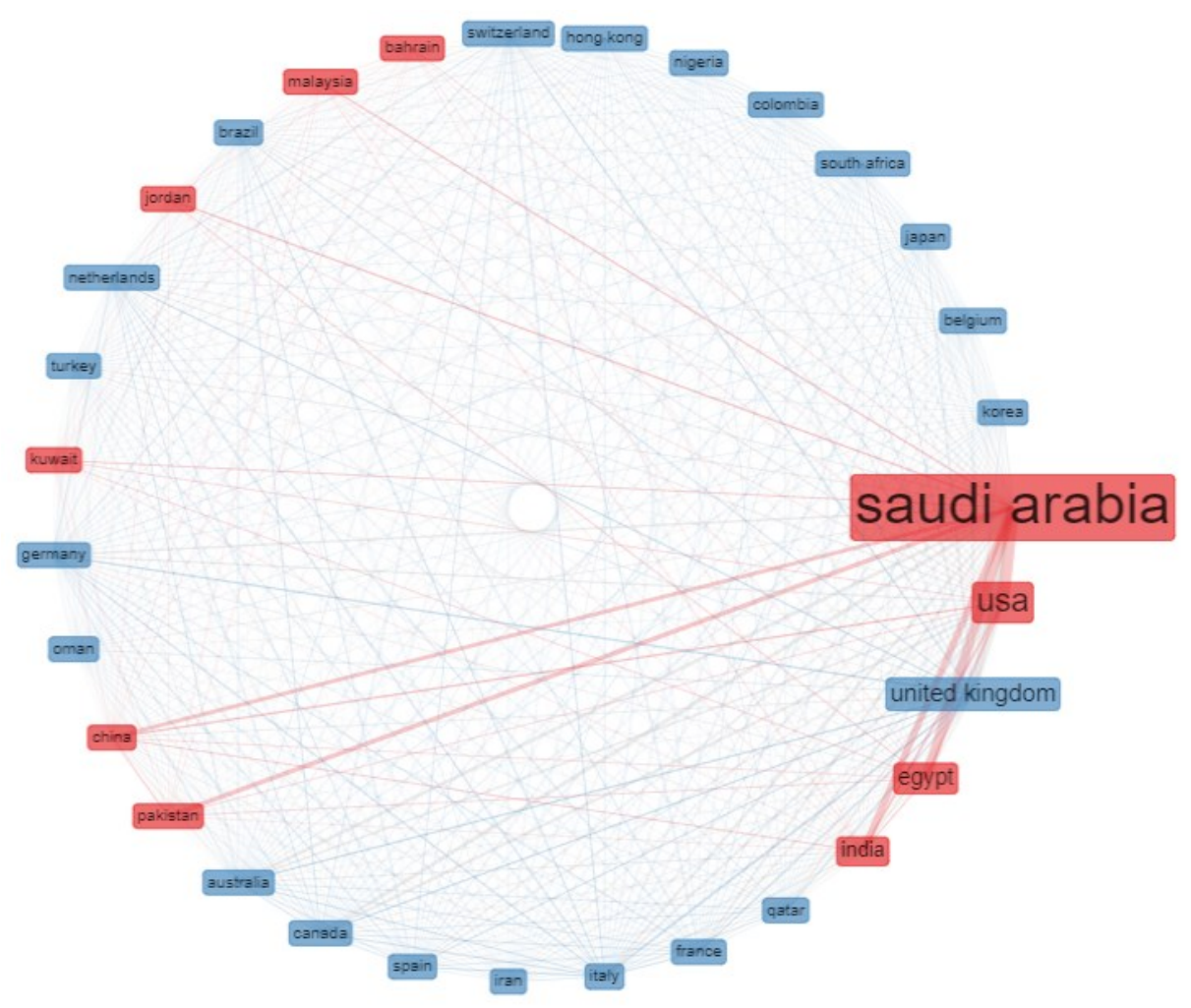

Fig. 13. Collaboration network of the most active countries

Furthermore, we considered the collaboration between the universities as well as shown in Figure 14. Three main clusters were recognized: cluster 1, represented in red, consisted of the King Saud bin Abdulaziz University for Health Sciences (KSAU-HS), Saudi Arabia, United Arab Emirates University, UAE, and King Abdullah International Medical Research Center (KAIMRC), Saudi Arabia. It is pertinent to note that KAIMRC is located with KSAU-HS.

Cluster 2 is the biggest one and is shown in blue color and contains primarily Saudi Arabian universities and institutions like King Saud University, King Abdulaziz University, Imam Abdulrahman bin Faisal University, King Faisal University, Jazan University, Taif University, King Khalid University, Qassim University, Umm alQura University, Taibah University, Jouf University, Princess Nourah bint Abdulrahman University, and King Saud University medical city. There are other universities from the GCC region in Group 2, such as the University of Sharjah, UAE, Qatar University, Kuwait University.

Group 3 contains universities and institutions such as the Ministry of Health, Alfaisal University, King Faisal Specialist Hospital and research center, Johns Hopkins Aramco healthcare from Saudi Arabia, and other US institutions like Indiana University School of Medicine and Emory University. 


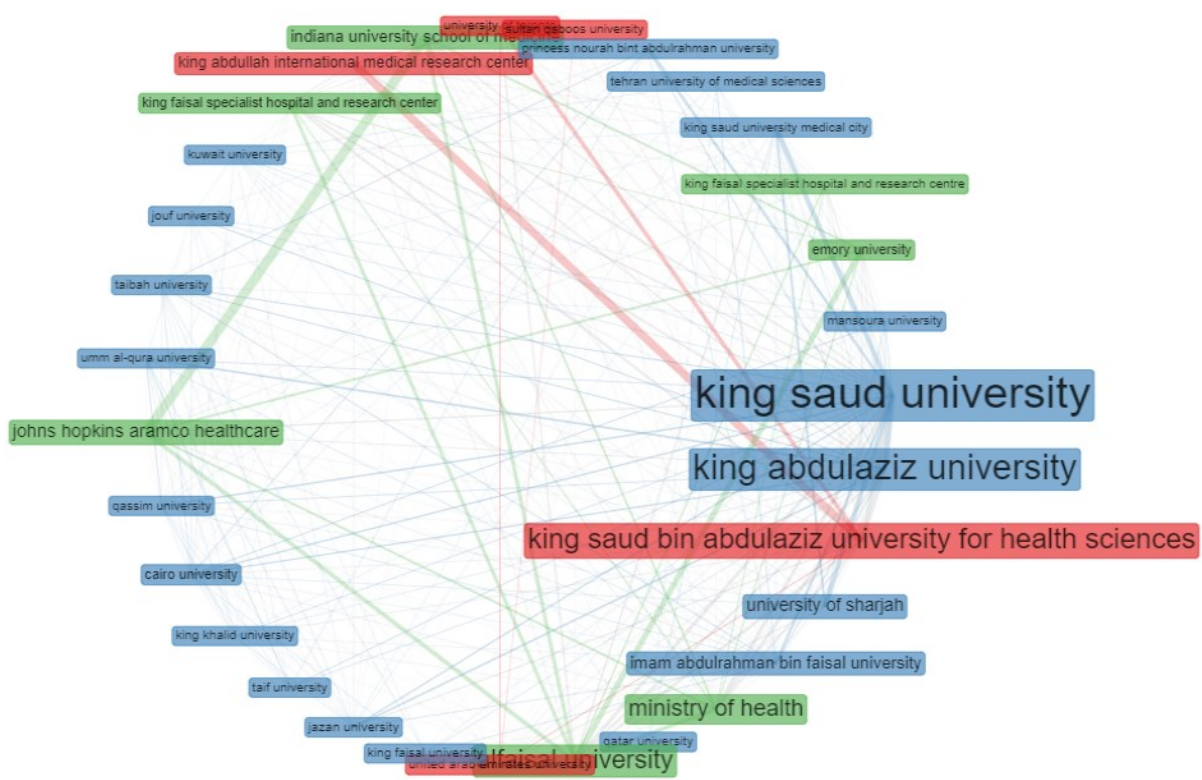

Fig. 14. Collaboration network of the most active institutions

Figure 15 illustrates the collaboration network of the leading researchers. Seven clusters were identified as compared to two for countries and three for the institutions. The researchers in the first clusters are Memish, Z.A., Al-Tawfiq, J.A., Assiri, A., and Alqahtani, S.A., and are shown in red color. We have ordered the researchers in line with the PageRank. Similarly, the most prolific authors in cluster 2 are Rabaan, A.A., and Sah, R., and are shown in blue. The third cluster includes Azhar E.I., Hassan A.M., El-Kafrawy, and others. The fourth cluster is a small one and contains only two researchers as shown in dark violet: Halwani, R. and Hamid, Q. Four, five, and two researchers are found in cluster five (orange), six (light brown), and seven (shown as light violet), respectively. 


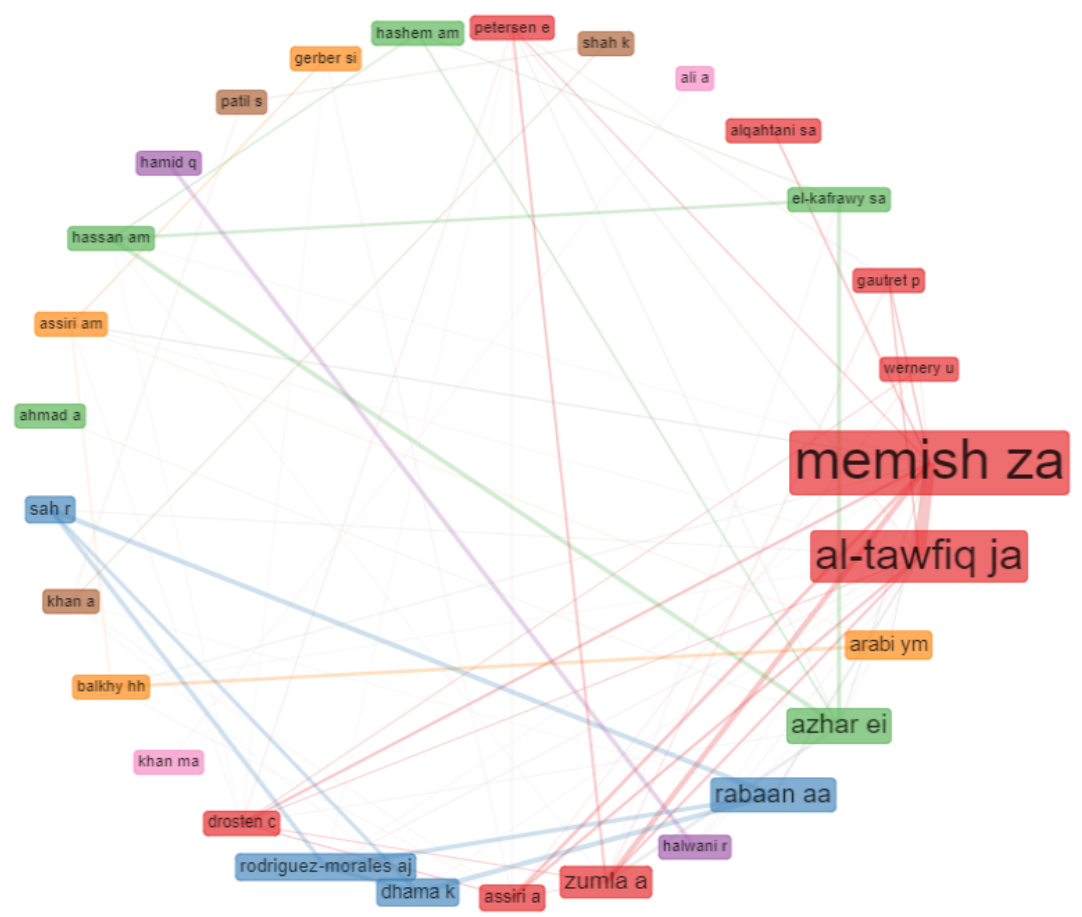

Fig. 15. Collaboration network of the most active researchers

Figure 16 shows the historical direct citation network. It represents the key research conducted between the years 2015-2021. We can observe that the most prolific papers were written by Omrani et al. in 2015, Al-Tawfiq et al. in 2016, and Rabaan et al. in 2017.

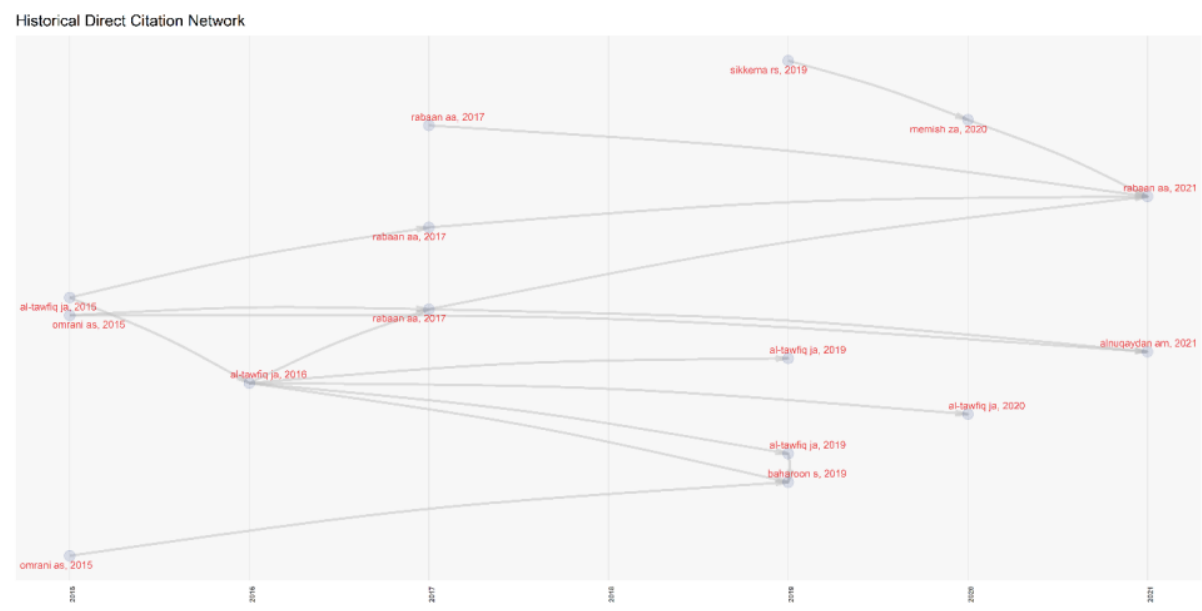

Fig. 16. Historical direct citation network from 2015 to 2021 
The word cloud acquired from the titles of the research papers is displayed in Figure 17. The most common word was found to be covid- having a frequency of 2598 . Words like coronavirus, pandemic, and Saudi followed this word, having a frequency of 796, 781, and 517, respectively. The other words have a frequency of less than 2800 .

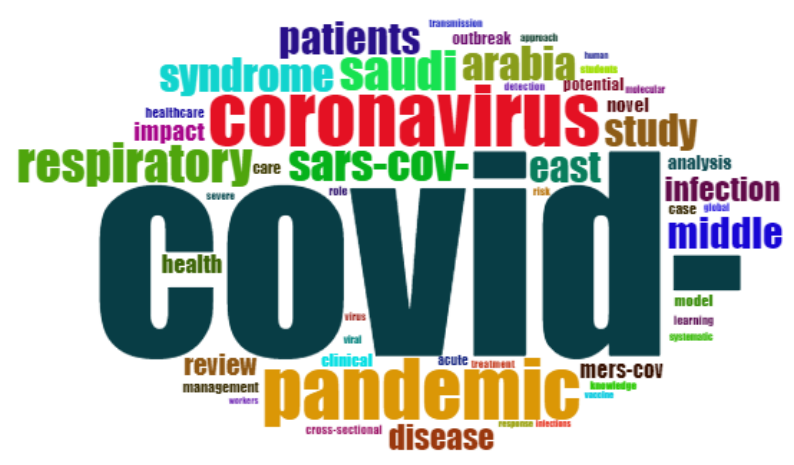

Fig. 17. Word cloud obtained from the titles of the research papers

Figure 18 shows the word growth for the keywords supplied by the authors over the years. The most remarkable growth is observed for the word COVID-19, followed by Sars-CoV-2 and Coronavirus.

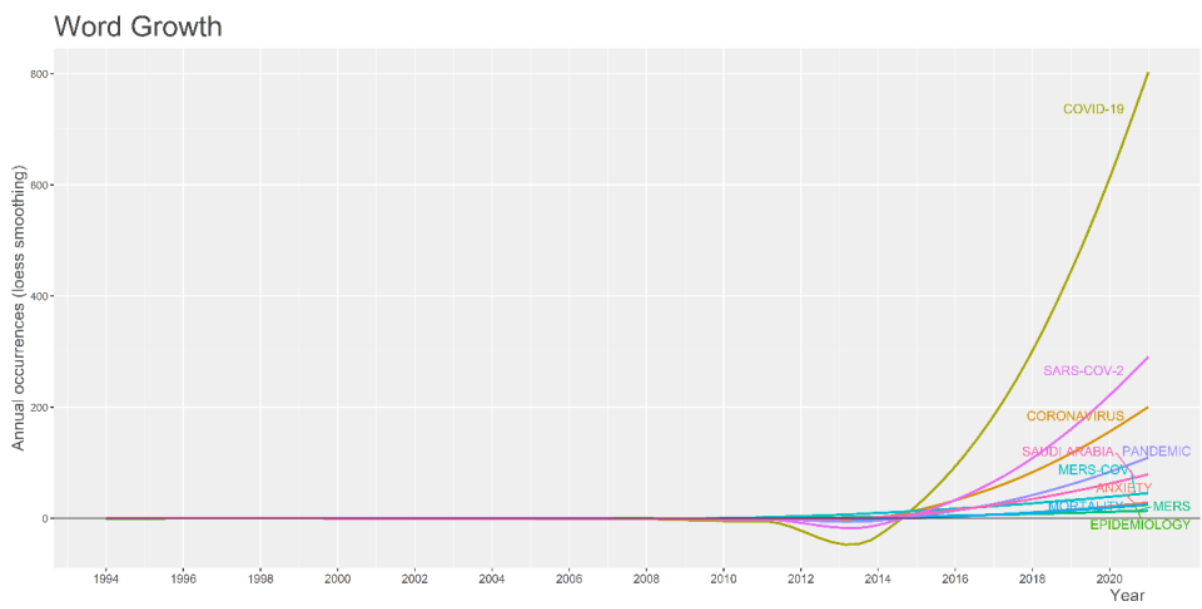

Fig. 18. Word growth by authors' supplied keywords from 1994 to 2021

Figure 19 shows the trending topics since 2013. The words covid-19, human, sarscov-2, epidemiology, viruses, coronavirus disease 2019, male and female are primarily observed in 2020 and 2021. On the other hand, MERS appeared more often in 2015 and appeared again in 2018 and 2019. This coincided with the reemergence of MERS 
cases. Similarly, the word camel(s) was observed in 2014, 2016 (dromedary), 2017, and 2018 (Camelus).

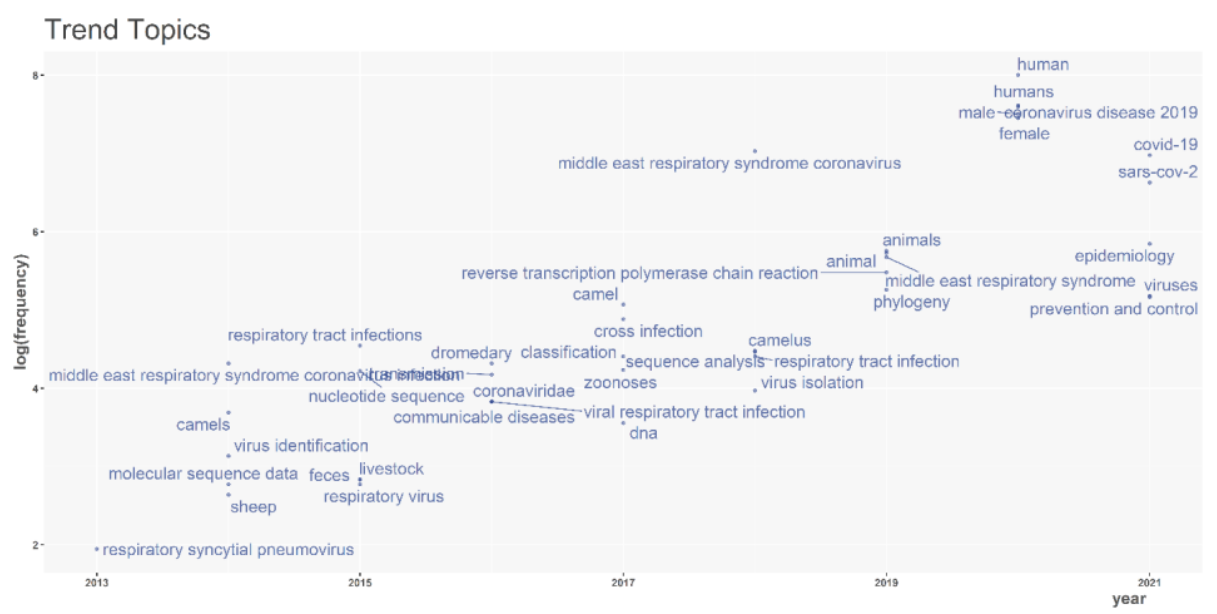

Fig. 19. Trending topics from 2013 to 2021

A thematic map helps analyze four types of themes according to the quadrant they are located. The themes in the upper right quadrant are the motor themes and depict high centrality (relevance) and density. Themes located in the lower-right quadrant are considered basic ones and have high centrality but lower density. The primary themes are critical for the research field. The emerging or declining themes are described in the lower-left quadrant and have lower centrality and density. These themes are considered peripheral. Lastly, niche themes are shown in the top-left quadrant. These themes have low centrality but high density. Figure 20 shows the thematic map for the keywords chosen by the authors. Deep learning appears as a niche theme. It has been observed that deep learning, a relatively new field in machine learning, is being applied in much research related to coronavirus. On the other hand, Saudi Arabia appears as a motor theme since most of the coronavirus research conducted in the GCC region is from this country. 


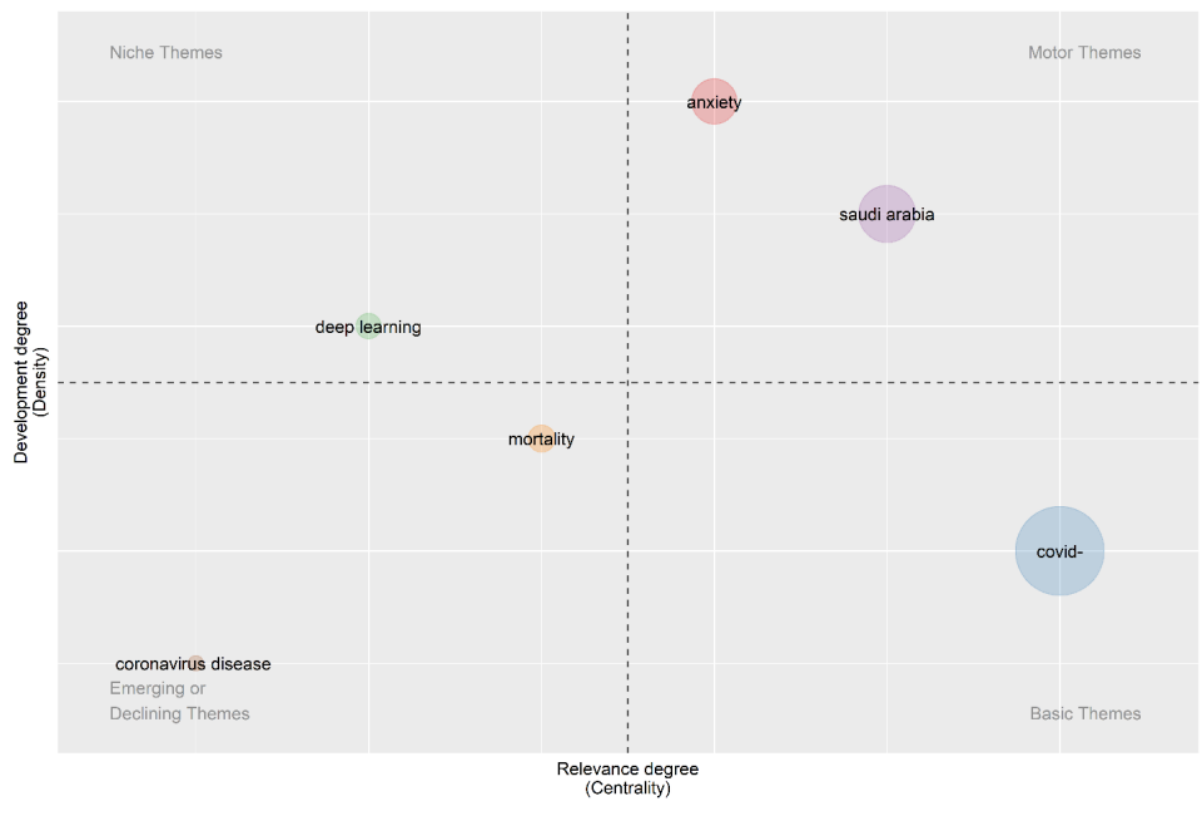

Fig. 20. Thematic map for the keywords chosen by the authors

Comparing our research work with previous works, we observed that the Journal of Virology got the maximum citations (37309) according to Danesh and GhaviDel [5]. However, according to our research, the most cited source is the New England Journal of Medicine, which got only 4025 citations. The vast difference in the number of citations could be attributed to our research being limited to the GCC region. Comparing our research with Zyoud [3], both found King Saud University and King Abdulaziz University the two most active institutions in coronavirus research. However, he focused on the entire Arab world, whereas we restricted ourselves to the GCC region. Similarly, as shown in Fig. 13, our research found the most active collaboration of Saudi Arabia with the USA, China, Egypt, India, and Pakistan, considering the countries outside the GCC. Zyoud found almost a similar behavior, i.e., Arab countries mostly collaborated with the USA, UK, India, Pakistan, and Italy.

\section{Conclusion and future work}

In this paper, we study the coronavirus publications in the Gulf Cooperation Council countries through bibliometric assessment. Four thousand two hundred eightyeight papers are retrieved from the Scopus database written during the last 28 years published in 1429 sources. The bibliometric data are processed using Biblioshiny. A bulk of the publications are in the form of articles and reviews. The most cited journal is the New England Journal of Medicine which has an impact factor of 74.699. Most of the publications are from 2020 and 2021, corresponding to the COVID-19 pandemic. The most cited country is Saudi Arabia, and the most active universities are King 
Saud University and King Abdulaziz University. A very strong collaboration was also observed between Saudi Arabia and countries like the USA, Egypt, India, and Pakistan. Memish, Z.A. wrote the most papers, followed by Al-Tawfiq, J.A. The trending topics such as covid-19, human, sars-cov-2, epidemiology, and viruses are identified in 2020 and 2021. Lastly, deep learning is observed as a niche theme that corresponds to its usage in many recent research works. In the future, we plan to perform similar research on the Web of Science database and make a detailed comparative analysis.

\section{References}

[1] Worldometers.info, Aug 10, 2021. Dover, Delaware, USA.

[2] Guleid, F.H., Oyando, R., Kabia, E., Mumbi, A., Akech, S., and Barasa, E. (2021). A bibliometric analysis of COVID-19 research in Africa. BMJ Global Health, 6(5): e005690. https://doi.org/10.1136/bmjgh-2021-005690

[3] Zyoud, S.H. (2021). The Arab region's contribution to global COVID-19 research: Bibliometric and visualization analysis, Globalization and Health, 17, Article no. 31. https://doi.org/10.1186/s12992-021-00690-8

[4] Gong, Y. et al. (2020). Early research on COVID-19: A bibliometric analysis. The Innovation, 1:2. https://doi.org/10. 1016/j.xinn.2020.100027

[5] Danesh, F., Ghavidel, S. (2020). Coronavirus: Scientometrics of 50 years of global scientific productions. Iranian Journal of Medical Microbiology, 14(1): 1-16. https://doi.org/10.30699/ijmm.14.1.1

[6] Zyoud, S.H. (2016). Global research trends of Middle East respiratory syndrome coronavirus: a bibliometric analysis. BMC Infectious Diseases, 16, Article ID 255. https://doi.org/10.1186/s12879-016-1600-5

[7] Farooq, R.K., Ur Rehman, S., Ashiq, M., Siddique, N., Ahmad, S. (2021). Bibliometric analysis of coronavirus disease (COVID-19) literature published in Web of Science 20192020. Journal of Family \& Community Medicine, 28(1): 1-7. https://doi.org/10.4103/ jfcm.jfcm $332 \quad 20$

[8] Kagan, D., Moran-Gilad, J., Fire, M. (2020). Scientometric trends for coronaviruses and other emerging viral infections. GigaScience, 9(8): 1-17. https://doi.org/10.1093/ gigascience/giaa085

[9] Lou, J., Tian, S.-J., Niu, S.-M., Kang, X.-Q., Lian, H.-X. et al. (2020). Coronavirus disease 2019: a bibliometric analysis and review. European Review for Medical and Pharmacological Sciences, 24(6): 3411-3421. https://doi.org/10.26355/eurrev_202003 20712

[10] Wang, L.L., Lo, K., Chandrasekhar, Y., Reas, R., Yang, J. et al., (2020). CORD-19: The COVID-19 open research dataset. Proc. 1st Workshop on NLP for COVID-19 at ACL 2020.

[11] Zyoud, S. H. and Al-Jabi, S. W. (2020). Mapping the situation of research on coronavirus disease-19 (COVID-19): a preliminary bibliometric analysis during the early stage of the outbreak. BMC Infectious Diseases, 20, Article no. 561. https://doi.org/10.1186/s12879$\underline{020-05293-\mathrm{Z}}$

[12] Ho, Y.-S., Fu, H.-Z., and McKay, D. (2021). A bibliometric analysis of COVID-19 publications in the ten psychology-related Web of Science categories in the social science citation index. Journal of Clinical Psychology, Online. https://doi.org/10.1002/jclp.23227

[13] Chahrour, M. et al. (2020). A bibliometric analysis of COVID-19 research activity: A call for increased output. Cureus Journal of Medical Science, 12(3): e5357. https://doi.org/ $\underline{10.7759 / \text { cureus. } 7357}$ 
[14] Luo, J. (2021) Forecasting COVID-19 pandemic: Unknown unknowns and predictive monitoring. Technological forecasting and social change, 166, Article ID 120602. https://doi.org/10.1016/j.techfore.2021.120602

[15] Zaki, A.M., van Boheemen, S., Bestebroer, T.M., Osterhaus, A.D.M.E., Fouchier, R.A.M. (2012). Isolation of a Novel Coronavirus from a Man with Pneumonia in Saudi Arabia. The New England Journal of Medicine, 367: 1814-1820. https://doi.org/10.1056/ nejmoa1211721

[16] Hui, D.S. et al. (2020). The continuing 2019-nCoV epidemic threat of novel coronaviruses to global health - The latest 2019 novel coronavirus outbreak in Wuhan, China. International Journal of Infectious Diseases, 91: 264-266. https://doi.org/10.3410/f.737216219. 793577187

[17] Raj, V.S. et al. (2013). Dipeptidyl peptidase 4 is a functional receptor for the emerging human coronavirus-EMC. Nature, 495: 251-254. https://doi.org/10.1038/nature12005

[18] Rodriguez-Morales et al. (2020). Clinical, laboratory and imaging features of COVID-19: A systematic review and meta-analysis. Travel Medicine and Infectious Diseases, 34: 101623. http://doi.org/10.1016/j.tmaid.2020.101623

[19] Assiri, A. et al. (2013). Epidemiological, demographic, and clinical characteristics of 47 cases of Middle East respiratory syndrome coronavirus disease from Saudi Arabia: a descriptive study. Lancet Infectious Diseases, 13(9): 752-761. https://doi.org/10.1016/S14733099(13)70204-4

[20] Assiri, A. et al. (2013). Hospital Outbreak of Middle East Respiratory Syndrome Coronavirus. The New England Journal of Medicine, 369: 407-416. https://doi.org/10.1056/NEJMoa1306742

[21] Zumla, A., Chan, J.F.W., Azhar, E.I., Hui, D.S.C., Yuen, K.-Y. (2016). Coronaviruses drug discovery and therapeutic options. Nature Reviews Drug Discovery, 15: 327-347. https://doi.org/10.1038/nrd.2015.37

[22] Alhazzani, W., et al. (2020). Surviving Sepsis Campaign: guidelines on the management of critically ill adults with Coronavirus Disease 2019 (COVID-19). Intensive Care Medicine, 46(5): 854-887. http://doi.org/10.1007/s00134-020-06022-5

[23] De Groot, R.J., et al. (2013). Middle East respiratory syndrome coronavirus (MERS-CoV): announcement of the Coronavirus Study Group. Journal of Virology, 87(14): 7790-7792. https://doi.org/10.1128/jvi.01244-13

[24] Van Boheemen, S., et al. (2012). Genomic characterization of a newly discovered coronavirus associated with acute respiratory distress syndrome in humans. mBio, 3(6): e0047312. https://doi.org/10.1128/mbio.00473-12

\section{$7 \quad$ Authors}

Ali Mustafa Qamar received the B.E. degree (Hons.) in computer software engineering from the National University of Sciences and Technology, Pakistan, in 2005, the M.S. degree in computer science from University Joseph Fourier (UJF), Grenoble, France, in 2007, and the Ph.D. degree in computer science from the University of Grenoble, France, in 2010. He has worked as a Temporary Assistant Professor with UJF, from November 2010 to June 2011. He is currently an Assistant Professor with the Department of Computer Science, College of Computer, Qassim University, Saudi Arabia. His research interests include machine learning, data mining, deep learning, social networks, and information filtering (email: al.khan@qu.edu.sa). 
Rehan Ullah Khan received the B.Sc. degree in information systems from the University of Engineering and Technology Peshawar, in 2004, the M.Sc. degree in information systems in 2006, and the Ph.D. degree from the Vienna University of Technology, Austria, in 2011. He is currently an Associate Professor with the Department of Information Technology, College of Computer, Qassim University, Saudi Arabia. He is also the Director Research and Development at the College of Computer, Qassim University. His current research interest includes pattern analysis and its applications in multiple disciplines (email: re.khan@qu.edu.sa).

Suliman A. Alsuhibany received the M.Sc. degree in computer security and resilience and the Ph.D. degree in information security from Newcastle University, U.K. $\mathrm{He}$ is currently an Associate Professor with the Department of Computer Science, College of Computer, Qassim University, Saudi Arabia. He has published in some of the most reputed journals and conferences. His research interests include human aspects of security (e.g. the so-called "usable security"), CAPTCHAs, spam-filter, keystroke dynamics, and information security (email: salsuhibany@qu.edu.sa).

Article submitted 2021-09-06. Resubmitted 2021-10-05. Final acceptance 2021-10-13. Final version published as submitted by the authors. 